\title{
Perceptual Learning and Abstraction in Machine Learning : an application to autonomous robotics
}

\author{
Nicolas Bredeche*, Shi Zhongzhi**, Jean-Daniel Zucker*** \\ nicolas.bredeche@lri.fr,shizz@ics.ict.ac.cn, jean-daniel.zucker@lip6.fr
}

\begin{abstract}
This paper deals with the possible benefits of Perceptual Learning in Artificial Intelligence. On the one hand, Perceptual Learning is more and more studied in neurobiology and is now considered as an essential part of any living system. In fact, Perceptual Learning and Cognitive Learning are both necessary for learning and often depends on each other. On the other hand, many works in Machine Learning are concerned with "Abstraction" in order to reduce the amount of complexity related to some learning tasks. In the Abstraction framework, Perceptual Learning can be seen as a specific process that learns how to transform the data before the traditional learning task itself takes place. In this paper, we argue that biologicallyinspired Perceptual Learning mechanisms could be used to build efficient low-level Abstraction operators that deal with real world data. To illustrate this, we present an application where perceptual learning inspired meta-operators are used to perform an abstraction on an autonomous robot visual perception. The goal of this work is to enable the robot to learn how to identify objects it encounters in its environment.
\end{abstract}

Index Terms-Abstraction, perceptual learning, feature selection, machine learning, real-world data.

\section{Introduction}

At the beginning of the 1980's, important works such as Marr's still considered the early stage of perceptual processing in humans and animals to be mostly hardwired [1]. However, an impressive amount of work has been conducted on Perceptual Learning since the beginning on the 1990's showing that learning also takes place in the early stages of the brain and have to be considered as a key component for information processing [2].

Given the original definition from Gibson, Perceptual Learning corresponds to any relatively permanent and consistent change in the perception of a stimulus array following practice or experience with this array [3]. To make it simple, Perceptual Learning is the learning that takes place at the perceptual level (as opposed to Cognitive or Abstract Learning). In fact, Perceptual Learning should be considered both as an important standalone process and as a complementary process to abstract learning for any real or artificial system that deals with real-world data. Current investigation focus on natural vision, audition, somatosensory and even motor-control capabilities to name a few.

From the Artificial Intelligence viewpoint, perceptual learning should be considered as an active process that embeds particular abstraction, reformulation and approximation within the Abstraction framework [4] [5] [6]. The active process refers to the fact that the search for a correct data representation is performed through several steps.

A key point is that Perceptual Learning focus on lowlevel abstraction mechanism instead of trying to rely on more complex algorithm. In fact, Perceptual Learning can be seen as a particular abstraction that may help simplify a complex problem thanks to a more relevant representation. Indeed, the baseline of Abstraction, i.e. choosing the relevant data to ease the learning task, is that many problems in machine learning cannot be solved because of the complexity of the representation whatever the learning algorithm - in some specific cases, this is related to the phase transition problem [7].

This paper is intended as a short introduction to Perceptual Learning and its possible use within a Machine Learning framework to help reducing the computational complexity of some problems. Section II presents some well-known complexity issues in Machine Learning. Then, section III review how biological systems may cope with such complexity issues. Section IV present an approach to reducing complexity in Machine Learning thanks to Perceptual Learning inspired operators and pinpoints several tools available so as to implement such an approach. Finally, the last section describes a real world application for object identification by an autonomous mobile robot 
that rely on the approach described therein. This project resulted in a real world application where a mobile robot wanders in the corridor of our laboratory, gradually learning how to identify recurrent objects of the environment, using no a priori knowledge on the target objects.

\section{Problem setting : complexity issues in Machine Learning}

A key issue in Machine Learning (and AI) is the computational complexity of any given problem. While some problems may really belong to the intractable class and cannot be solved efficiently, there exists a great deal of problems that are characterized from the worst case analysis viewpoint and for which there may be tractable solutions (such as the well known travelling salesperson problems [8] or even real-world problems [9]).

Depending on the chosen representation, many problems can either easily be solved or easily be proved unsolvable. However, at the frontier between the two regions, there exists a small frontier known as the phase transition ${ }^{1}$ where it is very difficult to prove either solvability or unsolvability. In a similar way, such a phase transition has been shown to take place in Machine Learning by [7].

More generally, it means that the initial representation which is used must be carefully chosen in order to make learning possible and that an appropriate change of representation may turn into a problem that can be solved. Getting out of the phase transition have often been addressed by using bias shifts [10] in order to change the representation.

As for real world problem, such that dealing with complex visual data, it is intersting to note that learning is very difficult because of the amount and richness of the data. As a matter of fact, classic supervised learning algorithms would try to link raw data to high-level symbols in some sort of abstract learning task that cannot cope with such a low-level representation.

\section{The neurobiology viewpoint : bridging the gap between perception and cognition}

In neurobiology as well as in Machine Learning, learning has long been considered to take place only at the cognitive level, i.e. either being processed in cognitive regions of the brain or handled by complex machine learning algorithms. However, many tasks in real life require humans to perform learning at the perceptual level.

As a matter of fact, learning was considered at the unique cognitive level where identification and categorization should be processed. However, Perceptual Learning

\footnotetext{
${ }^{1}$ Originally a term used in Physics.
}

is used to identify letters as a low-level stimulus or to 2 quickly discriminate between close colours, objects or tastes that can make many categorization task possible. In fact, a major claim is that perceptual learning and cognitive learning strongly interact during any learning task.

Thus, perceptual learning mechanisms act as very specific bias shifts in order to turn real world raw sensory data into more comprehensive ones [11]. As a matter of fact, researchers concerned with perceptual learning do study how short- and long-term adaptations are performed in the early stages of the brain that are driven both by the environment (i.e. unsupervised perceptual learning) and the cognitive necessities (i.e. supervised perceptual learning). Hence, perceptual learning mechanisms act on the very nature of sensory data so as to provide a more relevant representation from the system's perspective.

A great amount of works exists in neurobiology that deals with Perceptual Learning mechanisms and models ranging from sensory learning (vision, hearing, olfaction, taste) to sensorimotor learning. Important aspects of these researches cover the plasticity of the neural systems [12], low-level mechanisms [13], models of perceptual learning [14], implications in top-down process in recognition [15] to name a $\mathrm{few}^{2}$. As an example, the importance of Perceptual Learning has also been shown in human for face recognition [16] and object recognition [17].

However artificial and natural perceptual systems strongly differ, we think that studying perceptual learning mechanisms should provide interesting insights regarding the design of efficient artificial perceptual systems that deals with similar problems.

\section{A Perceptual Learning Approach to Ab- straction}

In Artificial Intelligence, "abstraction" techniques have long been used in order to solve complex problems such as hierarchical planification [18] or problem solving [19]. In those works, the idea was to highlight relevant properties in order to ease the deduction task. In a similar way, Abstraction in Machine Learning focus on changing the representation for induction, hence the following definition [6]:

Abstraction : An abstraction is a change of representation within the same formalism that hides some details and preserves some relevant properties in order to make the initial problem simpler to solve . $^{3}$.

Abstraction theory has been introduced in Machine Learning by [4] so as to ease induction during the learning

\footnotetext{
${ }^{2}$ A very good and complete overview of current works and issues can be found in [2].

${ }^{3}$ Thus, any abstraction can be seen as an homomorphism between two representations.
} 


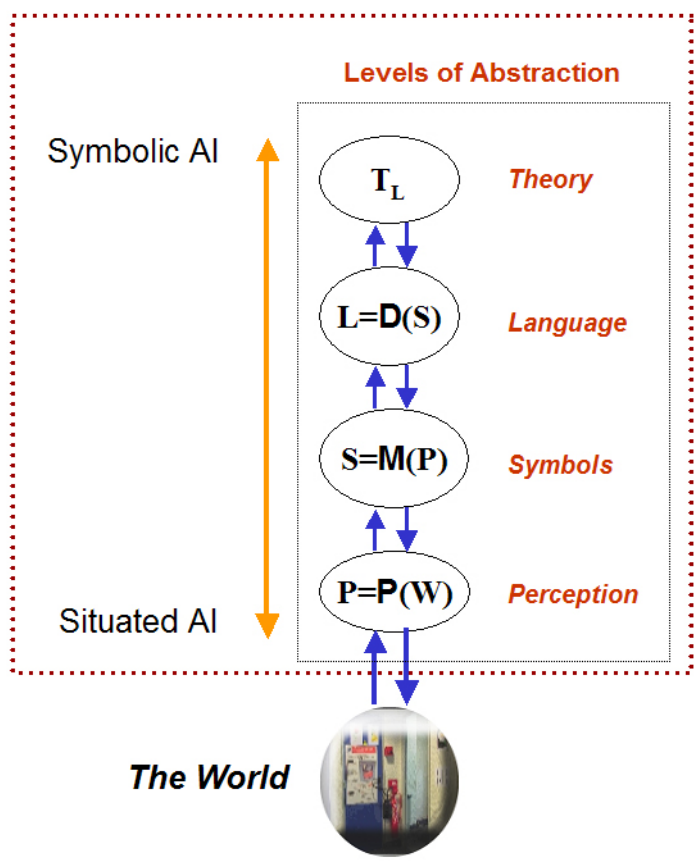

Fig. 1. The KRA model.

task by performing some kind of specific bias shifts to build a representation that highlights relevant data.

A general framework has been proposed that describes the several levels of abstraction for Machine Learning [5], [6] which roots can be traced to even more general Abstraction theory in AI [20], [21].

In the following, we describe how Perceptual Learning can be considered as a basis to perform a specific kind of abstraction at the perceptual level ("P-abstraction"). More precisely, we state that there exists a class of P-operators that can be inspired from Perceptual Learning in some specific context related to the nature of some problems.

Then, we review two very different tools that can serve as a basis to easily design and implement Perceptual Learning inspired operators.

\section{A. Perceptual Learning P-operators}

Figure 1 illustrates the KRA model (i.e. Knowledge Representation for Abstraction) proposed by Saitta and Zucker [22] that synthesizes the several levels of representation (Perception, Structure, Language and Theorem) and related abstraction processes. Between two contiguous levels, the abstraction process is performed thanks to Abstraction operators. This abstraction model can be seen as a somewhat universal model since it embeds previously known models concerning, for example, problem solving as well as planification.

As for Machine Learning, Most of the abstraction are situated at the first two levels. In fact, there have been ${ }^{3}$ a vast literature on Abstraction in ML with techniques concerning features [23], [24], [25], examples [26] or representation language [27] to name a few.

In the scope of this paper, we focus on abstraction operators that are first applied to raw data at the perceptual level, i.e. perception operator or P-operator which are defined as follow [6]:

P-operator : An abstraction P-operator $\omega$ corresponds to a transformation that takes into account a raw perception of the world $P_{g}(W)$ and performs an abstraction in order to provide a simpler perception $P_{a}(W)$ of the world $W$. the $\omega$ argument corresponds to the subpart of $P_{g}(W)$ that is actually transformed.

As a matter of fact, any P-operator may be applied on data of very different origin such as raw data from databases, texts, images, etc. Within the Abstraction framework, Perceptual Learning operators should then be considered as specific P-operators since they are applied at the sensory (i.e. perceptual) level. From now on, we shall refer to these operator as PL P-operators.

Of course, there is a great amount of P-operators that have nothing to do with Perceptual Learning as we have already seen (e.g. feature selection/construction). Thus, it is important to highlight the specificity of such PL Poperators.

Firstly, one should consider the data' source since Perceptual Learning may be useful to problems for which there exist real world counterparts encountered by living animals or humans. As an example, vision-related problems can be addressed with such operators since Perceptual Learning mechanisms have already proven to be relevant in real world situations. Nevertheless, such an inspiration should be conducted with care since we do not intend to make a precise model of a real perceptual system, i.e. experiments are intended to validate the efficiency of such approaches.

Secondly, Perceptual Learning may provide some help to build PL P-operators that can be used inside a given architecture, but it is not fitted if we intend to build the system's architecture itself. As a matter of fact and from the biological viewpoint, Perceptual Learning mechanisms should be considered only as underlying processes that take place in a greater architecture. To sum it up, studying real world Perceptual Learning mechanisms is but a privileged way to bootstrap the design of PL P-operators.

As a consequence, a PL P-operator could be simply defined as follows :

PL P-operator : A specific instance of $\boldsymbol{P}$-operator upon which a given heuristic may rely to solve a problem also addressed by real-world perceptual system.

In order to illustrate this, we will quickly described two well-known operators (among many others), namely 
unitization and differentiation that act as complementary processes. They are defined as follow (from [13]) :

- Unitization involves the construction of single functional units that can be triggered when a complex configuration arises (e.g. learning to identify letters or ideograms);

- Differentiation happens when stimuli that were once psychologically fused together become separated (e.g. learning to taste wines or to quickly identify nearlysimilar colours).

However these two operators are very specific to biological system, they may be considered respectively as a kind of generalization and specialization processes performed at the perceptual level (e.g. within a wrapper model). From the Machine Learning viewpoint, several abstraction operators have been proposed by some authors that are more or less related to the operators we have just described. An example is object or scene layout identification [28], [29] (see section V) where such operators are used so as to perform some kind of information filtering process on raw visual data.

\section{B. Elements of Machine Learning for Perceptual Learning}

In this subsection, we describe two possible uses of Machine Learning tools and algorithms in order to implement Perceptual Learning inspired operators. Of course, this list is far from exhaustive but addresses efficient and on the shelf tools for which there exists many comprehensive documentations as well as implementations that can be easily modified.

1) The wrapper and filter approaches: In Machine Learning, the abundant literature on feature selection shows that approaches fall in two broad categories: the wrapper and the filter approaches [30]. Intuitively, the wrapper model synthesized on figure 2 uses the performance of the learning algorithm as a heuristic to guide the abstraction. As it is an approach that attempt to learn from the learning process itself it is also referred to as a meta-learning approach. The filter model uses some specific ad hoc heuristic to choose one specific representation.

Originally, these two models were introduced to perform feature selection, i.e. choosing from a large set of features which are relevant for the task at hand. However, their use can be generalized in order to address one punctual change of representation using a handcrafted heuristic (filter model) or iterative changes of representation that rely on an accuracy guided search (wrapper model).

As a matter of fact, both the filter and wrapper models designate useful tools that can be used with a wide range of approaches. The wrapper model is particularly interesting

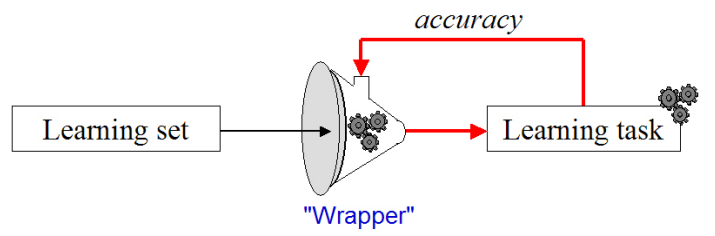

4

Fig. 2. The Wrapper model.

because thanks to the appropriate P-operators, it is possible to navigate within specific spaces of representations.

As a consequence, since the wrapper model makes it possible to build a representation to learn from, it is a perfect candidate for implementing Perceptual Learning inspired operators as long as it deals with the appropriate sensory data. For example, appropriate feature construction and destruction P-operators can be implemented so as to mimic the Unitization and Differentation perceptual learning mechanisms introduced before. In section $\mathrm{V}$, we will describe an architecture that combines the wrapper model and PL P-operators for object identification by a real-world mobile robot.

2) Constructive neural network: Since perceptual learning is related to the plasticity in the early stage of real or artificial systems, it is interesting to consider how it could be use within a very simple architecture that deals with low-level data. As a matter of fact, basic Artificial Neural Nets provide such a simple framework with simple units and it may be interesting to study if it's possible to apply specific growing and pruning operators in order to modify the very physical organization of such a network. In fact, there have already been several successful attempts at performing this [31], and various approaches have been proposed which, however, are not related to perceptual learning.

One of the most famous constructive neural networks is the Cascade correlation architecture [32]. This algorithm starts with a very small network (one input and one output), and then incrementally adds hidden nodes in order to minimize the overall error of the network (note that no node is ever erased). In order to do this, the algorithm tries to minimize the local error for each newly added node before adding a new one (i.e. it tries to correlate the new node output with the global output). Once again, the cascade architecture is often referred to as a meta-learning algorithm since it runs on top of the traditional network's mechanisms (backprop, etc.). The cascade correlation algorithm relies indeed on a rather simple method in order to add new nodes. However, it is known to solve problems that cannot be addressed with classic neural networks. Starting from this, one may wonder if it is possible to grow several nodes at a time using a different constructive algorithm. 
In fact, constructive neural networks offer a perfect opportunity to design constructive methods inspired from perceptual learning. As an obvious example, growing a new node in a network can be seen as some sort of differentiation process related to some stimuli. Beyond this simple example, it might be interesting to explore possible applications of this kind of perceptual learning mechanisms for the development of such a network.

Of course, one has still to take into account the danger of overfitting and other pitfalls well known from the NN community. However, implementing perceptual learning constructive algorithm might be a promising direction to explore. Note however that such an approach does not justify anything from the neurobiological viewpoint but instead may offers some interesting tracks to avoid difficulties related to the handcrafting of a network architecture (choosing the number of nodes, layers, etc.).

\section{Application of Perceptual Learning in Ma- chine Learning : an example application}

In this section, we illustrate the use of perceptual learning inspired approaches to address a problem relying on what was introduced previously. In order to do so, we demonstrate the use of operators that share some similarities with the simple unitization and differentiation perceptual learning operators for a batch supervised learning task.

We present here a work that has been conducted from 1999 to 2003 where the goal is to endow an autonomous mobile robot with the ability to learn how to identify objects from its environment. In this case, learning for object identification is achieved thanks to a wrapper approach that relies on biologically inspired operators to find the most relevant way to represent data.

\section{A. Perceptual learning for object identification by an autonomous robotics}

This research focus on learning how to identify objects in the corridor of our lab (e.g. human, robot, door, extinguisher, box, etc.). Here we will present our approach and some real world experiments that show the benefits of using perceptual learning inspired operators.

We are concerned with providing a PIONEER2DX autonomous mobile robot with the ability to learn how to identify objects in the real world. Because of the large amount of objects that the robot could be confronted to, it was not possible to rely on ad hoc object identification mechanisms. As a consequence, we have to endow the robot with the ability to cope with the intrinsic complexity of real world data and to learn from it - namely, to perform the grounding process [33], [34]. Thus, Abstraction
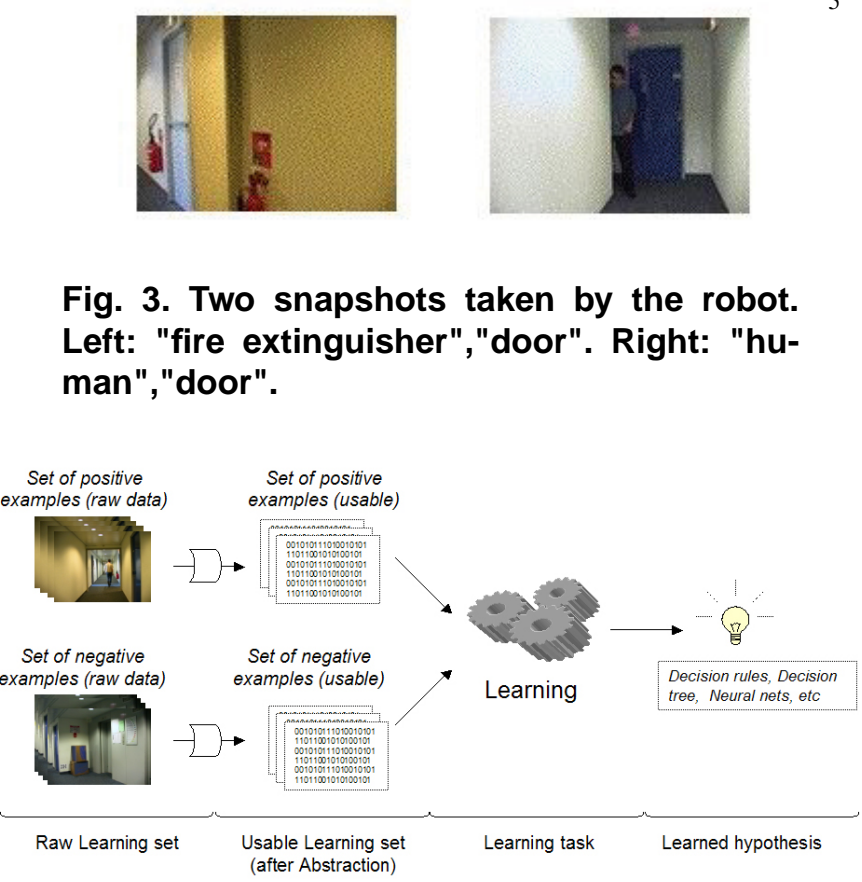

\section{Fig. 4. Abstraction preprocessing and learn- ing tasks.}

and Perceptual Learning are perfect candidates for our approach since we are dealing with complex images from the real world.

We used a PIONEER2DX mobile robot which behavior provides images thanks to its LCD video camera while navigating in the corridors of our lab. The images are $160 \times 120$ wide, with a 24 bits color information per pixel. Humans, robots, doors, extinguishers, ashtrays and other possible targets can be seen among the images as shown in Figure 3. All these possible targets appear in different shape, size, orientation and are sometimes partially occluded. Finally, each image is labelled with the names of the occurring targets during human-robot interactions.

A key aspect of the problem lies in the definition of the learning examples (i.e. the set of descriptions extracted from the images) used by the robot during the grounding process. As a matter of fact, a first step to any grounding process is to identify (relevant) information out of raw sensory data in order to reduce the complexity of the learning task. In practical, this means that given a set of a positive examples (images with the target object) and negative examples (same number of images, but without the target object), the goal is to learn how to classify a new image based on relevant invariant properties that can be found in at least one part of the image. The learning task is therefore characterized by a set of image descriptions 
and attached labels, as for a classic supervised learning task with an emphasis on the abstraction process which takes place before learning as illustrated in figure 4 .

\section{B. Initial Perceptual Representation}

We define the role of the robot's perceptual system as to extract abstract high-level percepts out of low-level percepts, such as a set of pixels, from the video camera. These high-level percepts provide a representation of the perceived world on which further computation will be based. They can be anything from sets of clustered colored regions to a matrix resulting from a Hough transform depending of the abstraction process. The choice of a representation is motivated by finding a good trade-off that reduces the size of the search space and the expressiveness of the high-level percepts.

As mentioned previously, the problem we consider is that of automatically finding a representation of a set of labelled images that is well adapted to the learning of concepts. In this set-up, We are interested in having a robot find by itself the good representation, so that, if the context changes or the concept to learn is different, it has the ability to discover by himself the good level of representation. We therefore consider the representation provided by the sensors as an initial representation.

From the robot's point of view, each pixel from the camera is converted into a low-level percept. In the initial image representation, where each pixel is described by its position $(\mathrm{x}, \mathrm{y})$, its hue (the tint of a color as measured by the wavelength of light), its saturation (term used to characterize color purity or brilliance) and its value (the relative lightness and darkness of a color, which is also refereed to as "tone"). The initial description of an image is therefore a set of 19200 (160 x120 pixels) 5-tuple $(\mathrm{x}, \mathrm{y}, \mathrm{h}, \mathrm{s}, \mathrm{v})$. Each image is labelled by symbols given by a human supervisor. The positive examples of a given concept (e.g., "presence of a fire extinguisher") to learn correspond to all images labelled positively for this concept. The negative examples are the images were the target concept does not appear.

The initial representation of images, consisting of hundreds of thousands of pixels, is clearly a too low-level representation to be used by Machine Learning algorithms. We have chosen the multiple-instance representation to represent information from the images. Within the multiple instance setting, objects are represented by bags of feature vectors. Feature vectors are also called instances and as in the traditional setting features may be numeric as well as symbolic. The size of a bag $b$ is noted $\sigma(b)$ and may change from one object to another. Its instances are noted $b_{1} \ldots b_{\sigma(b)}$. The multiple-instance representation is an in-between representation, more expressive than

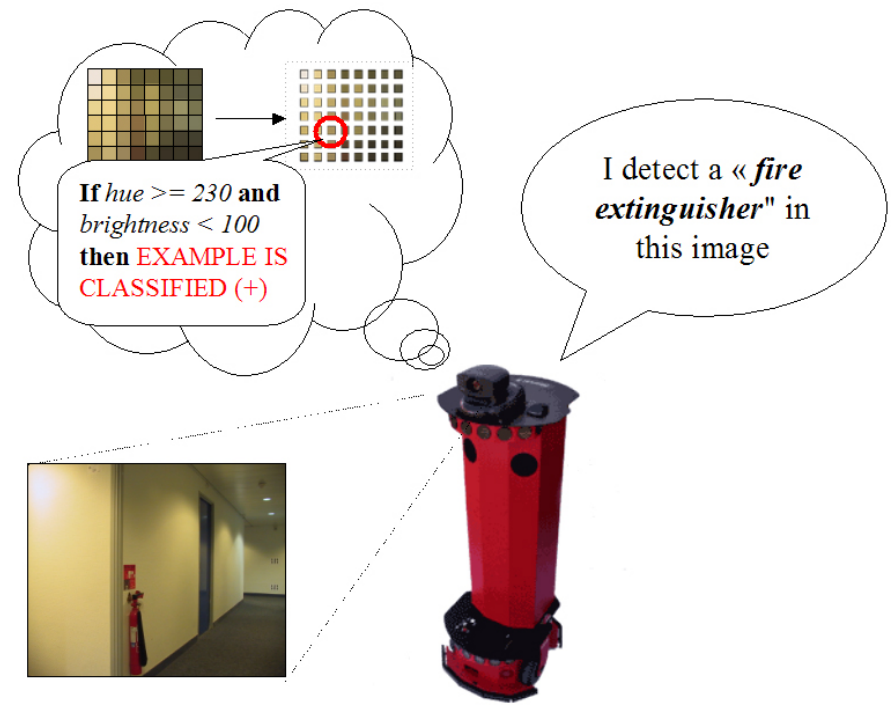

Fig. 5. Example of a multiple-instance hypothesis used for object detection.

feature-vector but for which efficient algorithms do exist, compared to algorithms used with a relational description. In practical, this means that given a set of examples (images with the target object) and counter examples (images without the target object), the goal is to learn how to classify a new image based on a specific invariant property that can be found in at least one part of the image (fig. 5 shows a practical example).

\section{Dimensions of abstraction}

In the perspective of automatically exploring the set of possible representations of an image, we propose to identify particular operators and to experiment with them. There are countless operators that could be applied to an image hoping for more accurate learning. Operators changing the contrast, the resolution, the definition are all possible candidates.

To improve the learning of concepts, we are interested in transformation that are abstractions in the sense that they decrease the quantity of information contained in the image [22]. The two main dimensions for abstraction that we studied are granularity (i.e. the resolution of the image) and structure (i.e. the smallest individually accessible portion of the image to consider, be it a pixel or a complex region which embeds several connected regions).

For each of these dimensions, we have defined an abstraction operator: respectively, associate (for granularity) and aggregate (for structure). The associate operator consists in replacing a set of pixels with a unique (mega)pixel that has for its $(\mathrm{h}, \mathrm{s}, \mathrm{v})$ values the average of the pixels that 


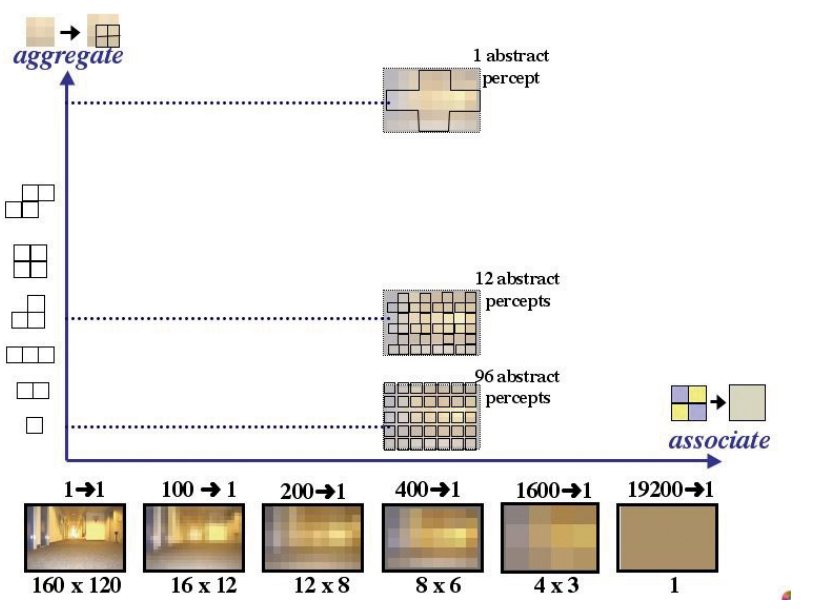

Fig. 6. Spaces of image representation.

were associated and corresponding standard deviations. This operator is a built-in operator for the robot as it corresponds to a particular sub-sampling - this operator will not be described in the scope of this paper. The aggregate operator consists in grouping a set of pixels to form a region or a pattern. This operation is also referred to as "term construction" in the literature [4]. The region does not replace the pixels it is composed of, and therefore the resolution or granularity of the image is not changed. What changes is the structure of the image. The aggregate operator may be either data-driven (this is the case for region growing algorithms) or model based. For already mentioned reasons of efficiency required by the use of a robot we have considered an aggregate operator that is applied to contiguous pixels forming a particular shape. Figure 6 depicts the space of representation changes associated to these two operators (the y-axis concerns the aggregate operator). Figure 7 depicts the main advantage of using such an aggregate operator. It is indeed possible to learn hypothesis that concerns several part of an image given a constrained structured pattern.

The aggregate operator acts as an abstraction function and provides specific image descriptions depending on the chosen parameters. Given a specific environment and object to be detected, the key issue lies in choosing the most relevant structure pattern (or s-percept) to break an image into parts. This is achieved by using Perceptual Learning meta-operators that acts on the very definition of what is the aggregate operator. As shown in figure 8, we rely on the Unitization and Differentiation PL-operators to devise the structure of an s-percept - each operator acting in an opposite direction. The idea is to be able to navigate through the space of aggregate operators as will be shown in the next paragraph.
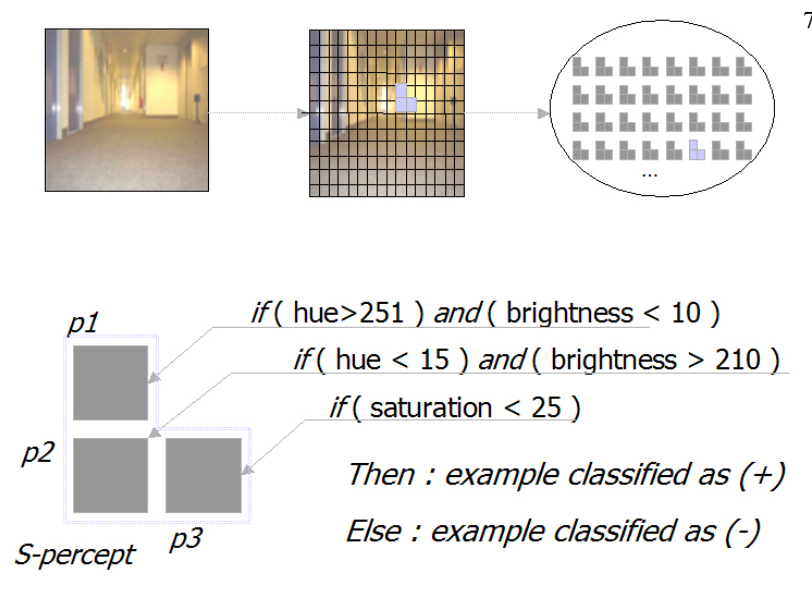

Fig. 7. Structured-percept-based hypothesis for object identification (example).

\section{Learning Grounded symbols}

With respect to the learning task described previously, a key issue is to analyze the impact of representation changes on learning. The main question is related to the choice of one operator and its parameters. We have used a specific wrapper approach to explore the spaces of abstraction described earlier. The PLIC system implements our approach for granularity and structure.

PLIC is a reformulation tool that acts as a wrapper according to given rules in order to find the best granularity and structure for describing the images. In the following experiments, PLIC acts as follow : given a granularity (e.g. $32 \times 24)$, an initial structure is chosen, and the image is reformulated in a multiple-instance representation using this structure; then, the concepts are learnt using this representation. Based on the results on cross-validation ${ }^{4}$ of the learning algorithm, a new structure is devised by PLIC using the aggregate operator along with the Unitization or Differentiation meta-operators. The search for a good structure is done starting from the simplest one (i.e., one mega-pixel at the chosen granularity) and exploring all the connected shapes of $k$ pixels before increasing $k$ only if the new structure provides better results. Figure 10 is a synthesis of this wrapper approach. The multiple instances rule learner RIPPERMI [35] was used on the descriptions obtained from these images with a ten-fold cross validation. Moreover, each experiment is repeated 10 times in order to get a good approximation of the results. RIPPERMI returns a set of rules that covers the positive examples. PLIC interacts with RIPPERMI in order to evaluate and create descriptions.

\footnotetext{
${ }^{4}$ a widely used data-oriented evaluation of the learning generalization error that consists in dividing the learning set into a learning set and a training set
} 


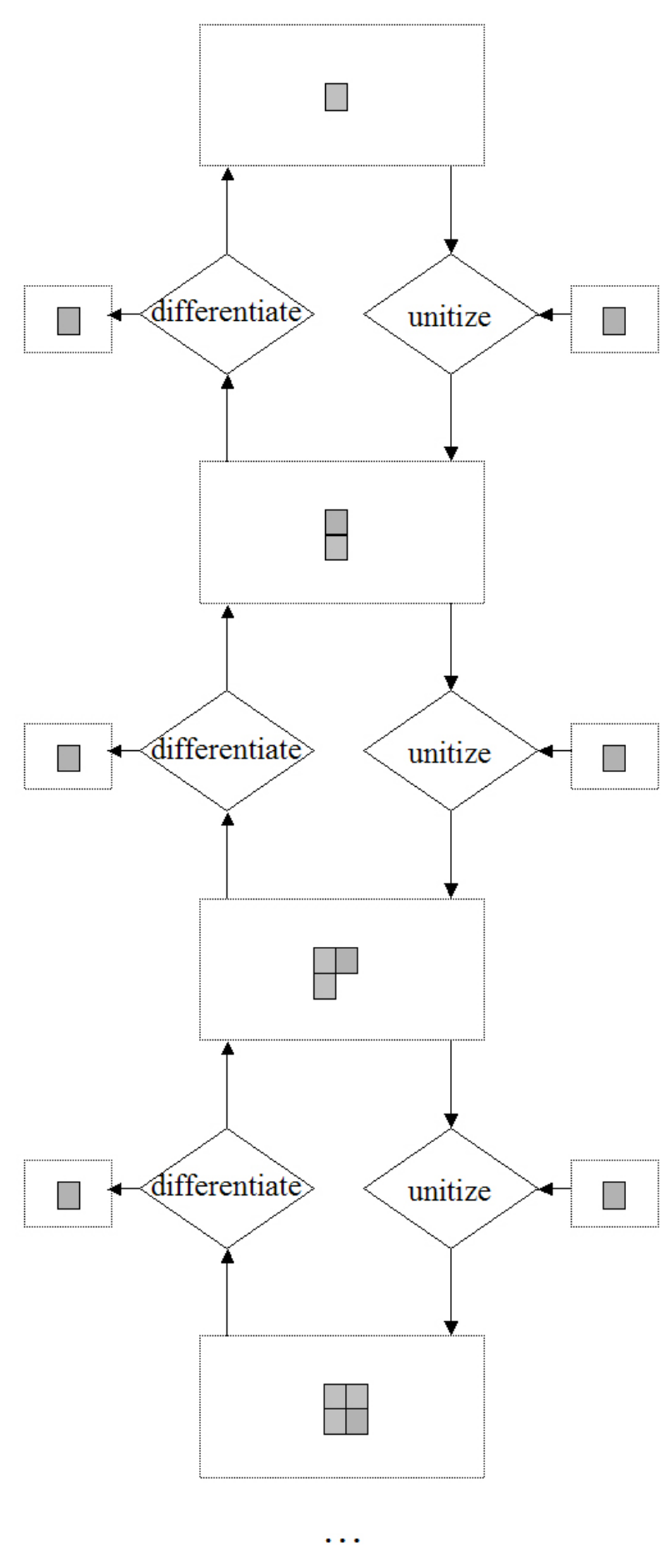

Fig. 8. Unitization and Differentiation metaoperators for Aggregation (example).

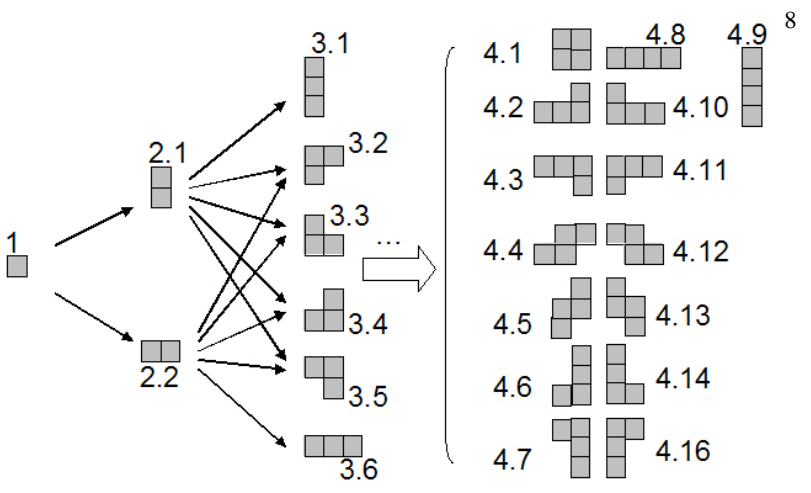

Fig. 9. Structural configurations explored by PLIC.

The experiments presented are based on the images acquired by a PIONEER2DX mobile robot. The attributes used for image description are : hue, saturation, value for each pixel, and hue, saturation, value and corresponding standard deviations for each high-level percept. The target objects are : box, fire extinguisher and human. For each experiment, 100 images were used (half of them being positive examples). PLIC considered a fixed granularity $(32 * 24)$ and different structures illustrated in figure 9 (up to 4 embedded elements).

Each structural configuration is applied from every single r-percept to generate the learning sets. The $32 \times 24$ resolution was chosen in order to show the potential of structural reformulation. Table I shows the best results achieved for each structural level of complexity.

Results from the experiments show that for all the objects, the highest accuracy is achieved by complex structural configurations (but not the most), which is not surprising. However the structural configurations are still quite simple, the identification accuracy for each object rose between 4.5 points (box detection) and 18.5 points (human detection) thanks only to structural information ${ }^{5}$. Figures presented at the end of the paper show a more indepth description of the accuracy achieved for the different concepts. The highlighted arrows show how the meta-operators where used to explore the space of possible representations.

Eventhough the impact of modifying the aggregation operator depends on the concept to learn (same as the association operator), structural reformulation is clearly an efficient way to improve classifiers for anchoring. For example, the following classifier was learnt from a reformulated dataset using the "3.3" s-percept :

- HYPOTHESIS: HUMAN.

${ }^{5}$ note that results differ from those we presented in [28] since a different learning set and features where used. 


\begin{tabular}{|c|c|c|c|}
\hline & "human" & "extinguisher" & "box" \\
\hline histogram & $67.4 \%$ & $64.6 \%$ & $79.9 \%$ \\
\hline level 1 & $60 \%$ & $76 \%$ & $77 \%$ \\
\hline level 2 & $70.5 \%(2.2)$ & $78 \%(2.2)$ & $77.5 \%(2.1)$ \\
\hline level 3 & $78.5 \%(3.5)$ & $80.5 \%(3.5)$ & $81.5 \%(3.2)$ \\
\hline level 4 & $75 \%(4.9)$ & $83 \%(4.15)$ & $80.5 \%(4.3)$ \\
\hline
\end{tabular}

TABLE I. object detection accuracy: best results for each structure level.

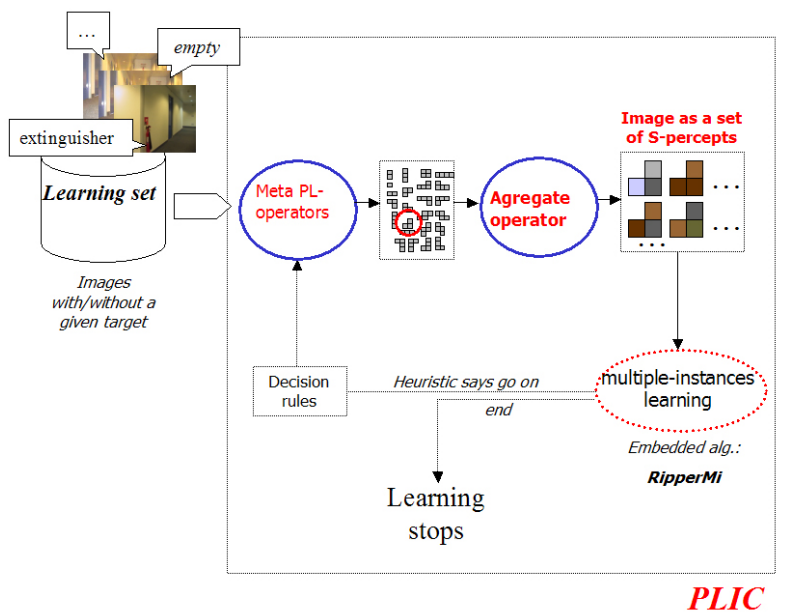

Fig. 10. PLIC - Perceptual Learning by Iterative Construction of percepts.

- TRUe :- P3VALUE $<=9$, P2SATURATION $>=27$.

- TRUe :- P2HUE $<=203$, P1SATURATION $<=3$, P3VALUe $<=165$.

- TRUE :- P3HUE $<=198, \mathrm{P} 1 \mathrm{X}>=6, \mathrm{P} 1 \mathrm{Y}>=2$.

- Default false.

(With P1, P2, P3 are the corresponding embedded rpercepts). This classifier gives a practical example that relations between the embedded percepts are taken into account since all percepts are considered in the rule.

\section{Discussion and conclusion}

In this paper, we argued that Perceptual Learning as studied in neurobiology may provide interesting and useful insights on how to build artificial perceptual systems.

Indeed, such an approach may prove highly reliable when it comes to artificial learning systems that deal with real world problem. As a matter of fact, it is known that humans and animals rely both on perceptual and cognitive learning so as to perform highly complex identification, recognition or even sensorimotor tasks. Thus, Perceptual Learning mechanisms help to better perform some kind of low-level abstractions.

From the Machine Learning viewpoint, the Abstraction framework is very much concerned with such perceptual
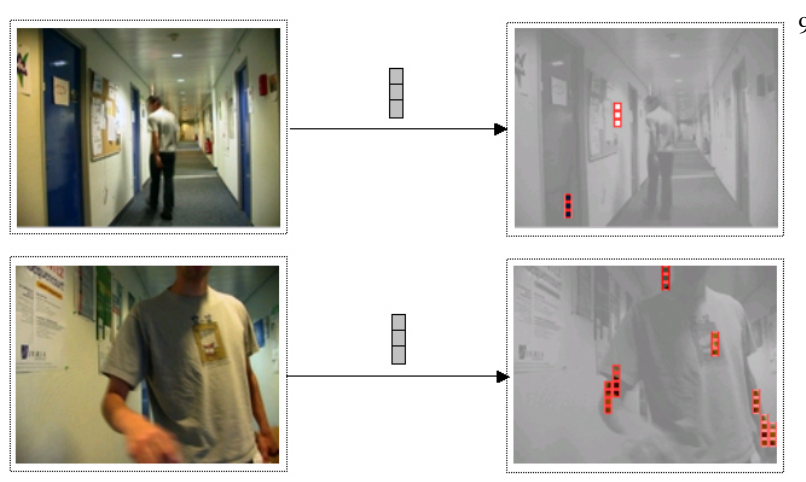

Fig. 11. Examples of learnt invariant properties.

learning mechanisms. In fact, some mechanisms can be implemented into artificial systems so as to provide relevant changes of representation regarding the tasks at hand.

We have described some useful Machine Learning tools as well as a real world experiment on object identification by an autonomous robotics. During our experiements, we showed that perceptual learning inspired abstraction operators can actually greatly enhance human identification accuracy for a mobile robot. As a matter of fact, such perceptual learning mechanisms are clearly relevant to situated robotics and AI since they allow to quickly adapt any perceptual system to the environment at hand.

This paper was intended as a short introduction to the study of Perceptual Learning in the field of AI and Machine Learning, providing an overview on complexity issues for natural and artificial systems as well as describing a specific approach, useful tools and a real world application. Within the Abstraction framework, we believe that operators inspired from what can be found in the environment may be a promising directions to build artificial yet efficient systems that address real world problems.

\section{References}

[1] D. Marr, Vision. Oxford: Freeman and Co., 1982.

[2] M. Fahle and T. Poggio, Eds., Perceptual learning. Bradford book, 2002.

[3] E. Gibson, "Perceptual learning," Annual review of Psychology, vol. 14, pp. 29-56, 1963.

[4] A. Giordana and L. Saitta, "Abstraction: a general framework for learning," in Working notes of the AAAI Workshop on Automated Generation of Approximations and Abstraction, Boston, MA, 1990, pp. 245-256.

[5] L. Saitta and J.-D. Zucker, "Semantic abstraction for concept representation and learning," in Symposium on Abstraction, Reformulation and Approximation (SARA98), S. i. P. b. AAAI), Ed., Asilomar Conference Center, Pacific Grove, California, 1998.

[6] J.-D. Zucker, "Changements de reprsentation, abstractions et apprentissages," Mmoire d'habilitation diriger des recherches, LIP6, Universit Paris 6, 2001. 
[7] A. Giordana and L. Saitta, "Phase transitions in relational learning," Machine Learning, vol. 41, no. 2, pp. 217-251, 2000.

[8] I. Gent and T. Walsh, "The TSP phase transition," Artificial Intelligence, vol. 88, pp. 349-358, 1996.

[9] I. P. Gent and T. Walsh, "Phase transitions from real computational problems," in Proceedings of the 8th International Symposium on Artificial Intelligence. 356-364, 1995.

[10] P. Utgoff, Machine Learning: An Artificial Intelligence Approach. Morgan Kaufmann, 1986, vol. 2, ch. Shift of bias for inductive concept learning.

[11] S. Edelman and N. Intrator, "Shift of bias for inductive concept learning," Perceptual learning, 2002.

[12] B. Zenger and D. Savi, "Plasticity of low-level visual networks," Perceptual learning, 2002.

[13] R. L. Goldstone, "Perceptual learning," in Annual Reviews of Psychology. 49:585-612, 1998.

[14] Y. Weiss, S. Edelman, and M. Fahle, "Models of perceptual learning in vernier hyperacuity," Neural Computation, vol. 5, no. 5, pp. 695$718,1993$.

[15] M. Herzog and M. Fahle, "Top-down information and models of perceptual learning," Perceptual learning, 2002.

[16] V. Bruce and M. Burton, "Learning new faces," Perceptual learning, 2002.

[17] G. Wallis and H. Bulthoff, "Learning to recognize objects," Perceptual learning, 2002.

[18] E. Sacerdoti, "Planning in a hierarchy of abstraction spaces," Artificial Intelligence, vol. 5, pp. 115-135, 1974.

[19] S. Amarel, "On representations of problems of reasoning about actions," Machine Intelligence, vol. 3, 1968.

[20] P. Nayak and A. Levy, "A semantic theory of abstraction," in Proceedings of the International Joint Conference on Artificial Intelligence (IJCAI-95), 1995, pp. 196-192.

[21] F. Giunchiglia, "Using abstrips abstractions : Where do we stand ?" Artificial Intelligence Review, vol. 13, pp. 201-213, 1996.

[22] L. Saitta and J.-D. Zucker, "A model of abstraction in visual perception," Applied Artificial Intelligence, vol. 15, no. 8, pp. 761776, 2001

[23] R. Kohavi and D. Sommerfield, "Feature subset selection using the wrapper method: Overfitting and dynamic search space topology," in International Conference on Knowledge Discovery and Data Mining, 1995.

[24] G. John, R. Kohavi, and K. Pfleger, "Irrelevant features and the subset selection problem," in Proceedings of the International Conference on Machile Learning, 1994, pp. 121-129.

[25] J. Wnek and R. Muchalski, "Hypothesis-driven constructive induction in aq17-hci - a method and experiments," Machine Learning, vol. 14, no. 2, pp. 139-168, 1994

[26] A. Blum and P. Langley, "Selection of relevant features and examples in machine learning," in Artificial Intelligence, vol. 97, 1997, pp. 245-271.

[27] J.-D. Zucker and J.-G. Ganascia, "Changes of representation for efficient learning in structural domains," in International Conference in Machine Learning. Bari, Italy: Morgan Kaufmann, 1996.

[28] N. Bredèche, Y. Chevaleyre, J.-D. Zucker, A. Drogoul, and G. Sabah, "A meta-learning approach to ground symbols from visual percepts," Elsevier's Robotics and Autonomous Systems journal, special issue on Anchoring Symbols to Sensor Data in Single and Multiple Robot Systems. S. Coradeschi and A. Saffiotti Eds. A paraitre., 2003.

[29] L. Hugues and A. Drogoul, "Pixel-based behavior learning," in Proceedings of the 15th European Conference on Artificial Intelligence (ECAI'02), 2002

[30] R. Kohavi and G. John, "The wrapper approach," in Feature Selection for Knowledge Discovery and Data Mining, H. Liu and H. Motoda (eds.), Kluwer Academic Publishers, pp33-50., 1998.

[31] E. Baum, "A proposal for more powerful learning algorithm," Neural Computation, vol. 1, pp. 201-207, 1989.

[32] S. E. Fahlman and C. Lebiere, "The cascade-correlation learning architecture," in Advances in Neural Information Processing Systems,

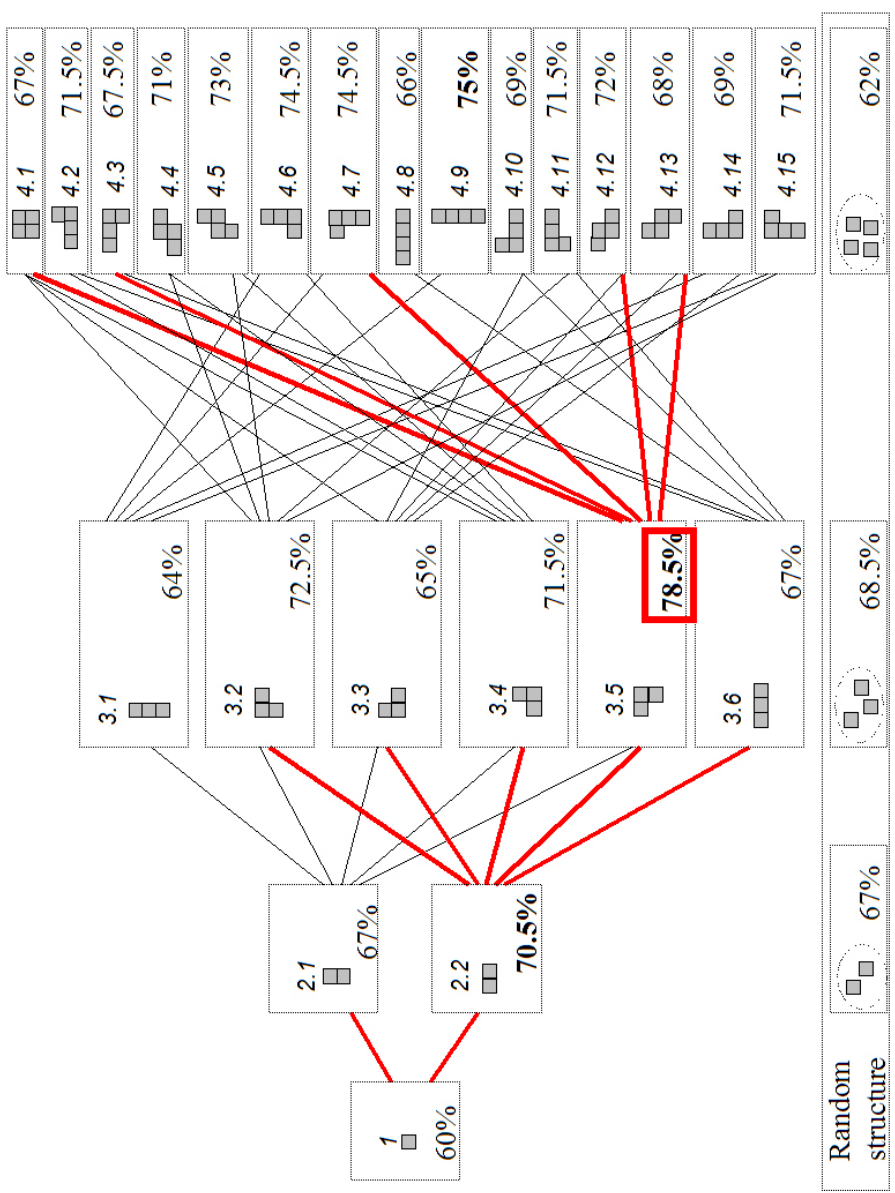

Fig. 12. Percepts achieved thanks to the Unitization and Differentiation meta-operatos target concept is human.

D. S. Touretzky, Ed., vol. 2. Denver 1989: Morgan Kaufmann, San Mateo, 1990, pp. 524-532.

[33] S. Coradeschi and A. Saffiotti, "Anchoring symbolic object description to sensor data. problem statement," Linkping Electronic Articles in Computer and Information Science ISSN 1401-9841, vol. 4, no. 9, 1999

[34] S. Harnad, "The symbol grounding problem," Physica, vol. D, no. 42, pp. 335-346, 1990.

[35] Y. Chevaleyre and J.-D. Zucker, "A framework for learning rules from multiple instance data," in Proc. European Conference on Machine Learning, 2001. 


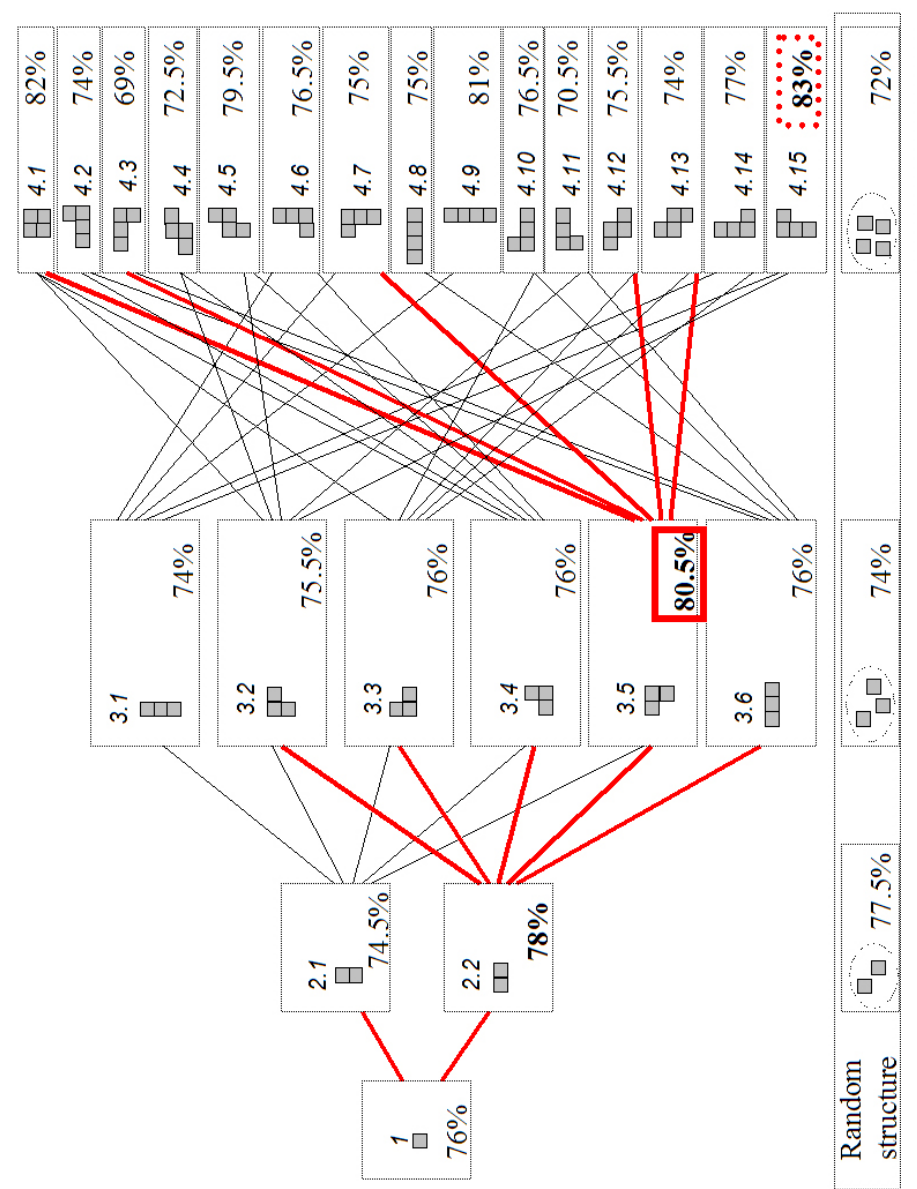

Fig. 13. Percepts achieved thanks to the Unitization and Differentiation meta-operatos target concept is fire extinguisher.

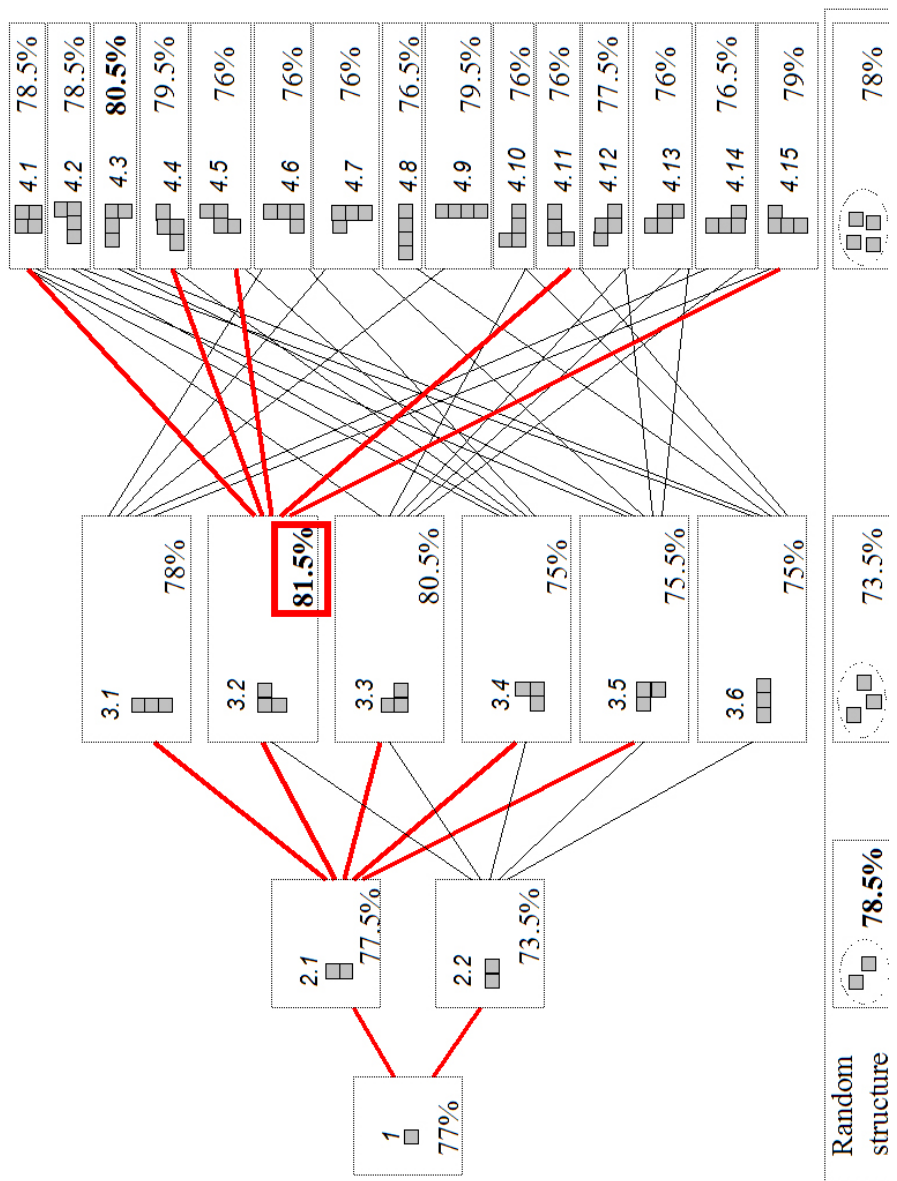

Fig. 14. Percepts achieved thanks to the Unitization and Differentiation meta-operatos target concept is box. 